\title{
Sleep and Actigraphy Research Trends: A Bibliometric and Keyword Network Analysis From 2004 to 2020
}

\author{
In Hye Park, RN, MSN ${ }^{1}$, Rahmi Qurota Aini, MEd ${ }^{2}$ \\ ${ }^{1}$ School of Nursing, University of Washington, Seattle, WA, USA \\ 2Department of Biology, Middle Tennessee State University, Murfreesboro, TN, USA
}

\author{
Received: September 5, 2021 \\ Accepted: October 7, 2021 \\ Correspondence \\ In Hye Park, RN, MSN \\ School of Nursing, University of Washington, \\ Box 357260, Seattle, WA 98195, USA \\ Tel +1-206-543-8736 \\ Fax +1-206-543-3624 \\ E-mail parkih@uw.edu \\ ORCID \\ In Hye Park \\ https://orcid.org/0000-0001-5180-1308 \\ Rahmi Qurota Aini \\ https://orcid.org/0000-0001-9743-5366
}

(c) This is an Open Access article distributed under the terms of the Creative Commons Attribution Non-Commercial License (https://creativecommons.org/licenses/by-nc/4.0) which permits unrestricted non-commercial use, distribution, and reproduction in any medium, provided the original work is properly cited.
Background and Objective As sleep is a predictor of quality of life, studying sleep health and measurement is important. Actigraphy as a physiologic measure based on monitoring an individual's movement has also been increasing in use as it has several advantages over traditional self-reports. This study aimed to report the research trends of sleep and actigraphy using a bibliometric and keyword analysis.

Methods Using topic search queries, this study collected data from 3955 publications published between 2004 and 2020 and listed in the Web of Science Core Collection. A text-mining unstructured data analysis was performed using author keywords and a bibliographic analysis.

Results Studies on sleep and actigraphy have been actively conducted in the fields of neurosciences and neurology but not in other fields. A gradual increase in publication and the steep increase in citations related to this topic occurring during the period considered. By clustering the author keywords, the analysis revealed five clusters: 1) monitoring physical activity and blood pressure, 2) measuring sleep quality, 3) insomnia and cognitive behavioral therapy for adolescents and children, 4) disorders and circadian rhythms, and 5) shift work. The leading research cluster has changed over the time.

Conclusions With the increase in the number of studies and citations on sleep and actigraphy over the past 16 years, the trend of sleep and actigraphy studies have shifted from a focus on sleeprelated psychiatric disorders to a focus on cognitive behavioral therapy for children and adolescents and, more recently, the measurement of sleep quality.

Sleep Med Res 2021;12(2):147-154

Keywords Actigraphy; Author keywords; Bibliometric; Research trend; Sleep.

\section{INTRODUCTION}

A culture that values healthy lifestyles more than previous generations did has led to interest in and research into better sleep [1]. Unfortunately, current studies have shown a gradual increase in sleep deprivation or sleep-related disorders across generations worldwide [2-5]. In fact, sleep clinic visits have been increasing [6]. In addition, the prevalence of insomnia has reached $6 \%-18 \%$ in the general population, although the exact rate depends on the definition of "insomnia" [7]. Insomnia negatively impacts physiological, neurocognitive, and psychological health [8], leading to increasing medical costs and, ultimately, sleep problems as a public issue [9]. Reflecting this trend, physiological diagnostic tools that assess sleep are attracting greater attention.

In particular, physiological sleep measurements have the advantages of tracking one's real-time sleep patterns and describing sleep stages that may be missed when using memory-dependent traditional sleep questionnaires/logs [10,11]. Actigraphy is a non-invasive, physiologic device that estimates sleep/wake patterns based on an individual's activity monitoring for weeks or months $[12,13]$. It can be placed on the wrist, trunk, or ankle, but is usually worn on the non-dominant wrist [14]. As Martin and Hakim [12] described, in the 1950s, a mechanical sensor-based de- 
vice was used to assess pediatric patients with psychiatric disorders; by the 1970s, it had been developed as a motor-activity monitor for clinical application with mainly psychiatric populations $[15,16]$. To date, it has been applied to various age groups in clinical studies. Previous studies have reported the high agreement of sleep parameters between actigraphy and polysomnography (PSG), a representative physiological sleep measure known as the gold standard, and also highlighted actigraphy's cost-effectiveness as it can be used for extended days with simplicity that does not need any specific sleep settings that could disturb one's sleep compared to PSG [17-19]. Currently, thanks to the small size and light weight of the evolved equipment, actigraphy is more actively used in research and clinical practice.

In studies to date, actigraphy has been used in healthcare-related fields such as medical and nursing to determine patients' sleep patterns in order to diagnose or treat sleep disorders or to assess the effectiveness of various treatments $[11,12]$. Some studies related to actigraphy have checked its validity [11]. However, few studies on recent research trends have comprehensively explored the purpose and scope of the use of actigraphy through specific literature searches such as a bibliometric review. A bibliometric analysis is a statistical method used to analyze the contents and trends of published studies through quantification [20]. The benefits of this type of analysis have been valuable in providing a timely, well-characterized review of a certain field of research that may ultimately help researchers guide and predict future research directions [21].

Considering the recent interest in sleep health, sleep-related industry, and quality of life, more demands to explore the research trends of actigraphy, an increasingly common method of sleep assessment, have emerged. Such research will help identify the changes in the utilization scope of actigraphy and its purpose of use, which have continually changed over time, and suggest diversity in insomnia care as well as predict the direction of further related research and clinical practice for assessing patients who complain about sleep problems.

In this study, we adopted a bibliometric method using previous studies to identify the contributions of the research on sleep and actigraphy based on publication keywords, the field of study, cited articles, and the findings of the community defined by the keywords. We analyzed the characteristics of actigraphy and sleep in research according to the frequency of keywords within publications and cluster classification through a bibliometric and keyword analysis to examine the current research trend and related research direction.

\section{METHODS}

\section{Data Collection}

The present study used data collected from the Web of Science
Core Collection using the keywords "actigraphy" AND "sleep" in topic search queries of research published between 1 Jan 2004 and 29 July 2020 . The initial search queries identified 3955 publications that contained both words in the title, abstract, and keywords. The Web of Science Core Collection includes scholarly journal publications, books, reports, and conferences indexed in SCI-EXPANDED, SCI, ESCI, and A-HCI. The data were exported with the full record and summary of search queries, such as publication year, research areas, and counties, for further analysis. The Web of Science Core Collection provides two kinds of keywords for each publication; author keywords were chosen rather than keywords plus for the authenticity of the research. However, 852 publications indicated to have missing keywords. Therefore, only 3103 publications contained the author keywords used in this analysis.

\section{Data Analysis}

The data were analyzed over three steps. First, a bibliography analysis was conducted by considering a summary of the number of publications and citations, the frequency of research area, and the most cited publications to examine the recent trends in sleep and actigraphy studies. Second, a text-mining unstructured data analysis of author keywords was conducted using the "Text Mining" package in R Software (https://www.r-project.org/).Data were transformed by removing unnecessary words such as "and," "the," common English stopwords, and punctuation, replacing similar meaning words as necessary (for example, "activities" to "activity"). The main keywords, "sleep" and "actigraphy," were excluded from the analysis to determine the frequency of keywords and their networks. In addition, term frequency (tf) and the term-document matrix ( $\mathrm{tdm}$ ) were created to calculate the number of times words occurred in a document and its matrix. Third, the clustering of networks was analyzed using the "iGraph" package (https://igraph.org/r/) by including only keywords that appeared more than 70 times, with weight less than $4(\leq 4)$ included for the network analysis. These keywords are representative enough to show the recent trends of interest in sleep health. The networks were generated based on the co-occurrence of keywords. Nodes in the networks represent the keywords connected at the edge with other keywords. The size of nodes in this network was set based on higher hub scores (Kleinberg's hub centrality scores). Using an optimized modularity score, clusters of networks were made according to the density of subgraphs. Furthermore, publication years in each cluster were calculated for further discussion.

\section{RESULTS}

\section{Most Frequent Research Area}

From the 94 research fields identified in the sleep and actigraphy research, $42.92 \%$ of the studies were categorized as neu- 
rosciences and neurology research. Following that, psychiatry constituted $14.64 \%$ of the research, while psychology and physiology research areas were ranked the third and fourth most frequent, with $8.77 \%$ and $7.21 \%$, respectively. The details of the most frequent research areas are presented in Table 1. Among the publications recorded in the Web of Science Data Collection, $50.34 \%$ of them came from the USA, followed by Australia (8.34\%), England (7.64\%), Canada (6.68\%), and Japan (5.31\%).

\section{Publication and Citation Trends}

The number of publications gradually increased from 75 to 557 publications between 2004 and 2019. In addition, the number of sum times cited increased exponentially, starting with 21 citations in 2004 and growing to 14241 citations in 2019. More information about the publication and citation trends is shown in Fig. 1.

\section{Most Cited References}

In order to determine the impact of research about sleep and actigraphy, the five most cited references are presented in Table 2. Publications from the clinical neurology and neurology area in 2004 led, with 1349 being cited in the Web of Science Core Collection. The most impactful research was found in Ohayon et

Table 1. Top 10 most frequent research area $(n=3103)$

\begin{tabular}{rlc}
\hline No & \multicolumn{1}{c}{ Research areas } & Records (\%) \\
\hline 1 & Neurosciences neurology & $1698(42.92)$ \\
2 & Psychiatry & $579(14.64)$ \\
3 & Psychology & $347(8.77)$ \\
4 & Physiology & $285(7.21)$ \\
5 & Life sciences biomedicine other topics & $216(5.46)$ \\
6 & General internal medicine & $192(4.86)$ \\
7 & Pediatrics & $187(4.73)$ \\
8 & Nursing & $175(4.43)$ \\
9 & Geriatrics gerontology & $150(3.79)$ \\
10 & Public environmental occupational health & $142(3.59)$ \\
\hline
\end{tabular}

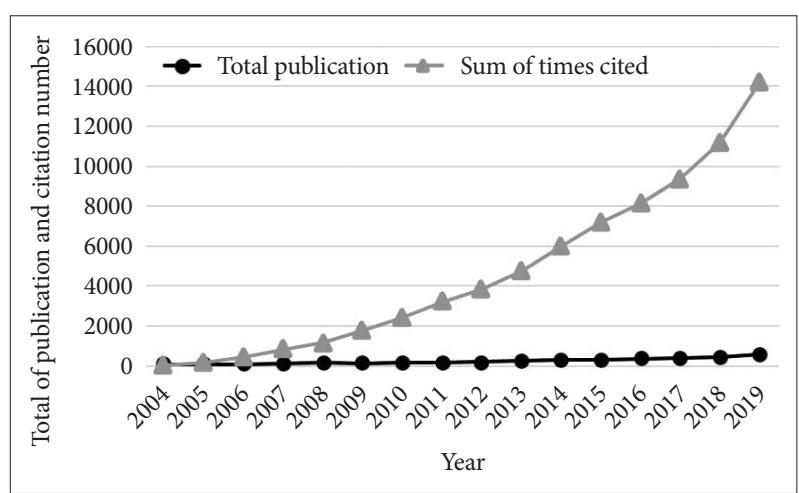

Fig. 1. Total of publication and citation number between 2004 and 2019 . al. [22], which aimed to identify sleep patterns in different age groups and sleep latency; the authors found that the percentages of stage 1 , stage 2 , and rapid eye movement sleep significantly change with age based on a meta-analysis of 40 years of literature. The second most impactful research by Lauderdale et al. [23] was categorized as public, environmental and occupational health research and sought to determine the degree of similarity between self-reported and wrist actigraphy-measured sleep duration. The third, fourth, and fifth most impactful publications were also categorized as clinical neurology and neurosciences research focused on disease and disorder in the central nervous system.

\section{Network and Cluster of Author Keywords of Publications on Sleep and Actigraphy}

The network analysis resulted in 48 important keywords related to sleep and actigraphy. The words repeated a minimum of 70 times, ranging from 5 to 41 connections. The main keywords, "sleep" and "actigraphy," were excluded in this analysis in order to see the research clustering around the topics of the main keywords. Table 3 summarizes the frequency of keywords and the centrality measure of the clustering network. The term "circadian" became the center of the network, with a higher hub score and frequency, followed by the words "rhythm" and "disorder." The keywords "quality" and "insomnia" also showed high connections in the network. In addition, a cluster analysis of author keywords around the topic of sleep and actigraphy generated five clusters. The visualization of the network is presented in Fig. 2, which includes 1) monitoring physical activity and blood pressure, 2) measuring sleep quality, 3) insomnia and cognitive behavioral therapy (CBT) for adolescents and children, 4) disorders and circadian rhythms, and 5) shift work.

\section{Trends in Cluster Groups}

As seen in Fig. 3, from 2004 to 2007, the studies in Cluster 4 (disorders and circadian rhythms, including bipolar disorder and sleep-wake disorder) were actively investigated the most. However, they were not significantly different from the number of studies in Clusters 2 and 3. From 2007 to 2012, the most investigated studies were in Cluster 3 (insomnia and CBT for adolescents and children), followed by Cluster 2 (measuring sleep quality), Cluster 4, Cluster 1 (monitoring physical activity and blood pressure), and Cluster 5 (shift work). Finally, from 2012 to 2019 , the number of studies increased in all clusters. Interestingly, the number of studies in Cluster 2 rose sharply, especially for about two years starting in 2017, showing an unrivaled trend of the rising keywords. Meanwhile, studies in Cluster 3 and Cluster 4 showed similar levels, followed by Cluster 1 and Cluster 5 during the period. 
Table 2. Top 5 most cited references in Web of Science Core Collection

\begin{tabular}{|c|c|c|c|c|c|}
\hline No & Times cited* & Article title & Publication year & Categories & Research areas \\
\hline 1 & 1349 & $\begin{array}{l}\text { Meta-analysis of quantitative sleep } \\
\text { parameters from childhood to old age in } \\
\text { healthy individuals: developing normative } \\
\text { sleep values across the human lifespan. }\end{array}$ & 2004 & $\begin{array}{l}\text { Clinical neurology; } \\
\text { neurosciences }\end{array}$ & Neurosciences \& neurology \\
\hline 2 & 727 & $\begin{array}{l}\text { Self-reported and measured sleep duration: } \\
\text { how similar are they? }\end{array}$ & 2008 & $\begin{array}{l}\text { Public, environmental \& } \\
\text { occupational health }\end{array}$ & $\begin{array}{l}\text { Public, environmental \& } \\
\text { occupational health }\end{array}$ \\
\hline 3 & 640 & $\begin{array}{l}\text { Practice parameters for the use of } \\
\text { actigraphy in the assessment of sleep and } \\
\text { sleep disorders: an update for } 2007 .\end{array}$ & 2007 & $\begin{array}{l}\text { Clinical neurology; } \\
\text { neurosciences }\end{array}$ & Neurosciences \& neurology \\
\hline 4 & 594 & $\begin{array}{l}\text { Recommendations for a standard research } \\
\text { assessment of insomnia. }\end{array}$ & 2006 & $\begin{array}{l}\text { Clinical neurology; } \\
\text { neurosciences }\end{array}$ & Neurosciences \& neurology \\
\hline 5 & 569 & $\begin{array}{l}\text { The role and validity of actigraphy in sleep } \\
\text { medicine: an update. }\end{array}$ & 2011 & $\begin{array}{l}\text { Clinical neurology; } \\
\text { neurosciences }\end{array}$ & Neurosciences \& neurology \\
\hline
\end{tabular}

*Times cited Web of Science Core Collection.

\section{DISCUSSION}

This study examined the trends of sleep and actigraphy studies using a bibliometric and keywords analysis based on the Web of Science Core Collection. In the findings, the studies about sleep and actigraphy were, as expected, most active in neuroscience or neurology. Given that neurology deals both directly and indirectly with sleep management, it can be inferred that physiological measurements may also be used to study related sleep physiology and sleep disorders. Furthermore, the results of the U.S.'s outstanding publication of sleep and actigraphy compared to other countries showed that researchers in the U.S. are leading the research and interest in the latest physiological sleep measures. The number of citations is traditionally used to indicate the impact of a particular study [24]. According to the findings of this study, the number of publications increased by about eight times and the sum of the citations increased by about 670 times, showing a steep increase in both interest in and impact of sleep and actigraphy research in the recent 16 years. In particular, a meta-analysis by Ohayon et al. [22], the most cited reference, summarized patterns of sleep with age has changed over 40 years and demonstrated that studies using actigraphy have been included in a variety of literature and cited by many researchers. The second most cited study, by Lauderdale et al. [23], compared selfreports and actigraphy, suggesting that researchers have more recently paid attention to similarities and differences in actigraphy using traditional self-reported methods, showing much interest in how sleep patterns can be used for diagnostic or therapeutic applications.

In addition, we examined the most used keywords around the topic of sleep and actigraphy based on a centrality measure in the network. The pivot words including high connections were circadian rhythms, disorder, sleep quality, and insomnia. This result was anticipated considering that actigraphy, an activity-based device, is used for the evaluation of sleep-wake rhythms and related disorders and insomnia.

Furthermore, the five clusters categorized in the cluster analysis of authors' keywords revealed the focus of the research over the past 16 years. The first cluster covered the topic of monitoring physical activity and blood pressure. It is not surprising that actigraphy is the unit sensor used to measure humans' activity and rest cycle. Blood pressure is also well-known to be influenced by one's activity and sleep as well as vice versa. One of the articles representing this cluster was published by Agarwal and Light [25], who explored the association of ambulatory blood pressure of sleep and physical activity. The second cluster focused on measuring sleep quality. In fact, various sleep studies have sought to measure one's sleep quality regardless of being healthy or not using physiologic measures like actigraphy. One example of such studies is Chen et al's [26] one that measured sleep disturbances, including quantifying sleep disorder breathing, short sleep duration, and poor sleep quality, and expanded the limit of sleep measuring research across different racial and ethnicities. In addition, Schwab et al.s [27] systematic review evaluating sleep quality among patients in an intensive care unit (ICU), which extended the use of actigraphy to the ICU. The third cluster covered insomnia and CBT for adolescents and children. Insomnia, one of the sleep disorders, is the main key feature in this network. CBT also became prominent in this cluster. CBT has been widely applied and recommended for sleep-related disorders [28,29]. Such representative non-pharmacology therapy can be used more for adolescents and children than other populations. A study by Palermo et al. [30] can be one of examples for this cluster, which explored the effects of CBT for insomnia in adolescents with physical or psychiatric comorbidities. The participants used self-reports and wrist actigraphy to measure sleep quality and behaviors, insomnia, psychological symptoms, and overall healthrelated quality-of-life outcomes. Disorders and circadian rhythms 
Table 3. Centrality measure of clustering network

\begin{tabular}{|c|c|c|c|c|c|c|}
\hline Cluster & Keywords & Frequency & Degree & Strength & Betweeness & Hubs \\
\hline \multirow[t]{7}{*}{1} & Activity & 293 & 35 & 675 & 36.50 & 0.395 \\
\hline & Physical & 124 & 24 & 316 & 48.17 & 0.159 \\
\hline & Monitoring & 99 & 12 & 159 & 4.95 & 0.045 \\
\hline & Pressure & 94 & 11 & 232 & 2.50 & 0.066 \\
\hline & Adult & 84 & 14 & 125 & 5.00 & 0.074 \\
\hline & Blood & 82 & 9 & 205 & 4.33 & 0.056 \\
\hline & Function & 74 & 9 & 90 & 1.33 & 0.054 \\
\hline \multirow[t]{17}{*}{2} & Quality & 298 & 41 & 610 & 19.33 & 0.229 \\
\hline & Polysomnography & 239 & 31 & 395 & 37.75 & 0.138 \\
\hline & Duration & 166 & 18 & 221 & 5.50 & 0.044 \\
\hline & Disease & 156 & 23 & 281 & 24.50 & 0.172 \\
\hline & Health & 120 & 25 & 213 & 55.07 & 0.062 \\
\hline & Apnea & 110 & 19 & 173 & 21.67 & 0.048 \\
\hline & Sleepiness & 104 & 19 & 185 & 8.83 & 0.077 \\
\hline & Stress & 104 & 12 & 112 & 21.28 & 0.045 \\
\hline & Syndrome & 102 & 17 & 159 & 12.67 & 0.089 \\
\hline & Time & 91 & 20 & 126 & 85.33 & 0.048 \\
\hline & Study & 85 & 15 & 120 & 10.00 & 0.040 \\
\hline & Variability & 78 & 17 & 131 & 26.42 & 0.057 \\
\hline & Index & 75 & 17 & 177 & 7.50 & 0.065 \\
\hline & Efficiency & 72 & 9 & 84 & 2.00 & 0.016 \\
\hline & Validation & 72 & 5 & 69 & 1.00 & 0.024 \\
\hline & Diary & 71 & 9 & 59 & 26.17 & 0.028 \\
\hline & Obesity & 71 & 11 & 103 & 1.33 & 0.054 \\
\hline \multirow[t]{14}{*}{3} & Insomnia & 348 & 38 & 728 & 12.08 & 0.301 \\
\hline & Child & 235 & 27 & 283 & 45.83 & 0.122 \\
\hline & Cognition & 197 & 31 & 473 & 27.58 & 0.193 \\
\hline & Adolescent & 183 & 28 & 296 & 51.03 & 0.108 \\
\hline & Behavior & 176 & 25 & 405 & 57.90 & 0.181 \\
\hline & Depression & 152 & 24 & 306 & 48.67 & 0.214 \\
\hline & Fatigue & 141 & 23 & 266 & 8.95 & 0.153 \\
\hline & Therapy & 117 & 21 & 368 & 14.08 & 0.184 \\
\hline & Disturbance & 98 & 17 & 167 & 13.83 & 0.106 \\
\hline & Cancer & 95 & 14 & 213 & 1.50 & 0.157 \\
\hline & Pain & 84 & 14 & 124 & 12.17 & 0.042 \\
\hline & Performance & 82 & 11 & 116 & 3.03 & 0.074 \\
\hline & Symptom & 72 & 12 & 96 & 2.33 & 0.038 \\
\hline & Chronic & 71 & 16 & 163 & 20.87 & 0.052 \\
\hline \multirow[t]{8}{*}{4} & Disorder & 448 & 38 & 983 & 18.67 & 0.657 \\
\hline & Circadian & 446 & 37 & 1227 & 7.17 & 1.000 \\
\hline & Rhythm & 417 & 36 & 1198 & 31.67 & 0.996 \\
\hline & Melatonin & 136 & 16 & 313 & 10.53 & 0.318 \\
\hline & Light & 105 & 19 & 296 & 34.45 & 0.279 \\
\hline & Bipolar & 88 & 10 & 224 & 4.00 & 0.253 \\
\hline & Sleep-wake & 88 & 10 & 156 & 2.50 & 0.178 \\
\hline & Dementia & 71 & 11 & 131 & 9.12 & 0.119 \\
\hline \multirow[t]{2}{*}{5} & Work & 79 & 15 & 199 & 7.58 & 0.099 \\
\hline & Shift & 71 & 15 & 191 & 11.92 & 0.090 \\
\hline
\end{tabular}




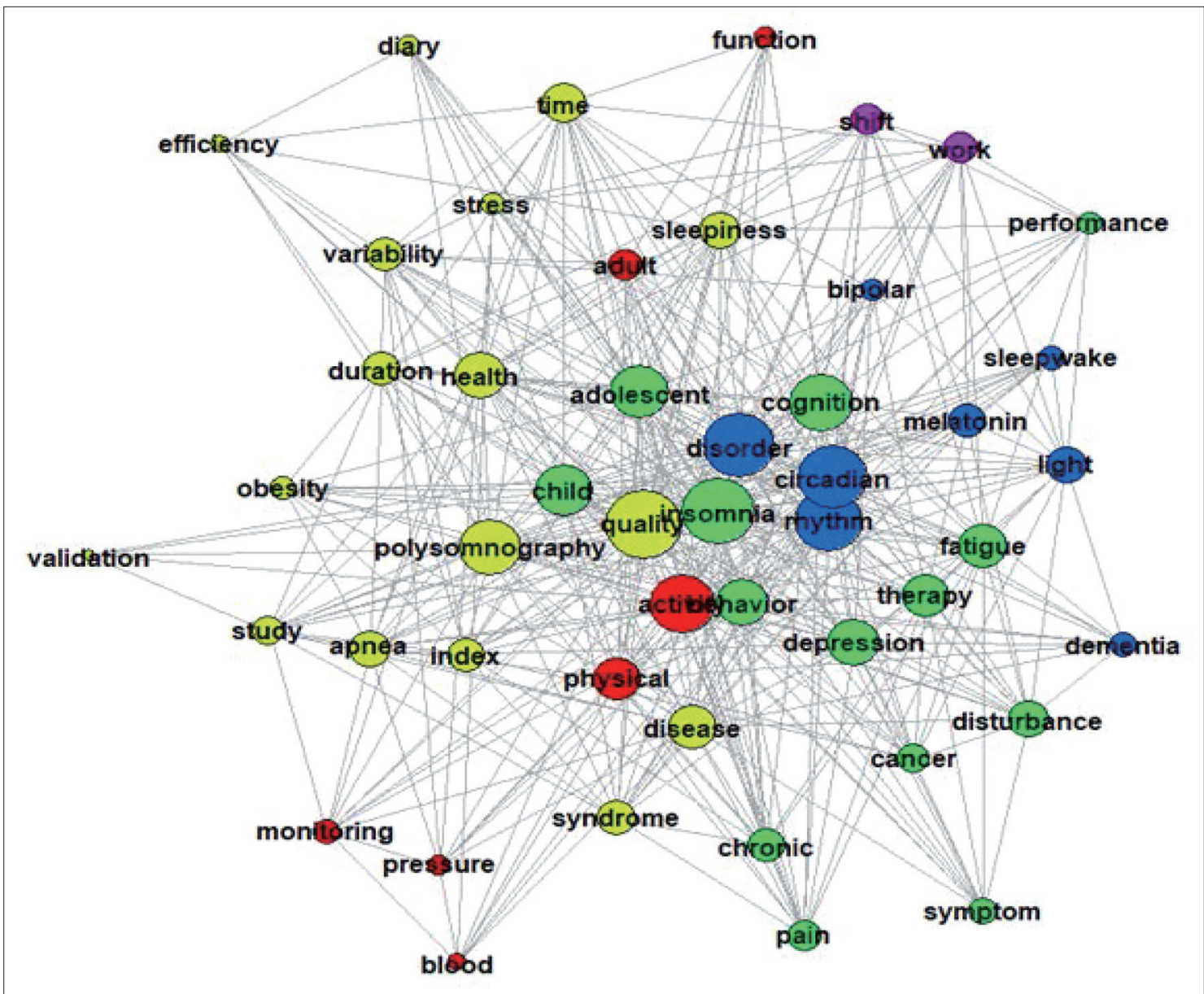

Fig. 2. Network and cluster analysis result. Red, Cluster 1; yellow, Cluster 2; green, Cluster 3; blue, Cluster 4; purple, Cluster 5.

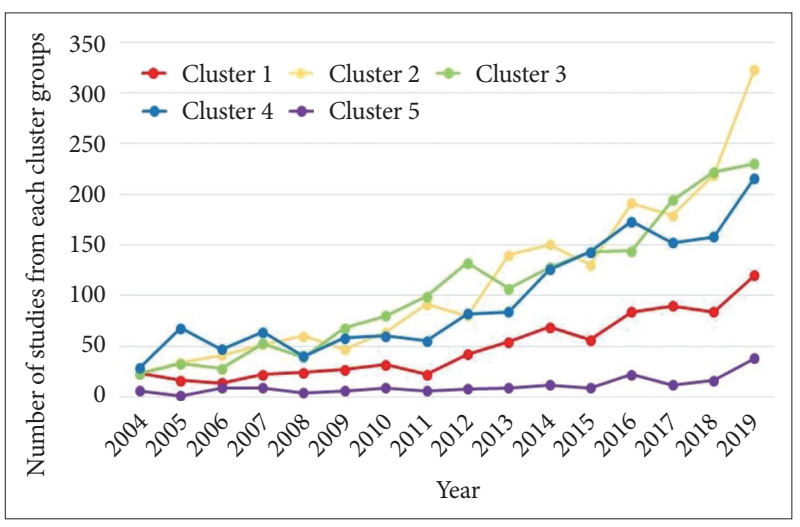

Fig. 3. Trend of keywords in each groups.

were main part of the fourth cluster, which broadly incorporated disorders, including sleep-wake and bipolar disorders. The second topic in this cluster, circadian rhythms, is a biological process that allows organisms to have sleep patterns. A representative study about this cluster presented by Esaki et al. [31] demonstrated that light exposure and the effect of blue-blocking were associated with sleep and circadian rhythms in patients with bipolar disorder. The fifth cluster represented sleep patterns and other effects among shift workers. Shift workers, especially nightshift workers, have different natural circadian rhythms than dayshift workers, so their disrupted sleep and treatment have often been discussed and researched [32]. For example, Heath et al. [33] investigated the association among diet, sleep, and mood in shift worker nurses. Another study by Mizuno et al. [34] explored sleep patterns of flight controllers of international space stations. Both studies measured the participants' sleep using actigraphy.

In the meantime, the leading research cluster has changed over time. First, between 2004 and 2007, actigraphy was widely used in Cluster 4 research related to psychiatric disorders. However, the differences in the number of studies between Cluster 4 and other groups were not significant, suggesting that actigraphy studies were similarly low across all clusters. About five years after 2007, Cluster 3 (insomnia and CBT for children) became the main focus, suggesting that actigraphy was widely used to identify the CBT effects on sleep problems before and after treatment, especially for children and adolescents in clinical practice. Finally, from 2012 to 2019, the number of studies from Cluster 2 (measuring sleep quality) also showed a significant increase in sleep and actigraphy research, which might reflect that actigraphy has been widely used for clinical or research purposes in the 
last seven years. It can also be inferred that the interest in and importance of sleep quality are being highlighted more as part of the quality of life, where the use of physiological measures is more active than before.

This study offers several methodological advantages. Previous studies on approaches to sleep quality analysis are directly related to several diseases and overall wellness [35]. In order to measure sleep patterns in a natural condition, devices such as actigraphy are known as an efficient method. However, the approach to measure sleep quality itself and the research of the actigraphy still lack a methodological standard that solidifies the outcome generalization [36]. The current study evaluates the current and previous trends of sleep and actigraphy studies in different fields. A combination of bibliometric analysis and semantic network analysis have been used to investigate systematic literature search about sleep and actigraphy from the abundance of information. The bibliometric analysis provided a quantitative analysis of written publications [37] whereas the semantic analysis highlighted the concept and scope in the network forms and perspective [38]. Using the central words and network classification as a result, the concept and scope of sleep and actigraphy studies from the most recent 16 years were defined. This methodology can introduce research that represents a defined scope and identifies the most impactful study in the scope of the field. The rapid growth of research using social network analysis in the early 2000s demonstrated this application embedded in bibliometrics and webmetrics [39]. The study of research trends using a keyword network analysis was used to understand literature in the huge quantitative scale. For example, the study conducted by Kho et al. [40] considered the use of "co-citation" and "co-words" to understand social formations in the organization. In the current study, 3103 publications were collected and analyzed to recognize the research direction and identify new topics [40]. Although previous studies have used a text network analysis, they have often focused on only one journal or a very limited period of time. In the end, the current study offered a more systematic literature review of sleep and actigraphy for a 16-year period.

\section{Availability of Data and Material}

The datasets generated or analyzed during the study are available from the corresponding author on reasonable request.

\section{Author Contributions}

Conceptualization: Park IH. Data curation: all authors. Formal analysis: all authors. Methodology: all authors. Supervision: Park IH. Validation: all authors. Visualization: all authors. Writing—original draft: all authors. Writing-review \& editing: Park IH.

\section{Conflicts of Interest}

The authors have no potential conflicts of interest to disclose.

\section{Funding Statement}

None.

\section{REFERENCES}

1. Williams SJ, Boden S. Consumed with sleep? Dormant bodies in consumer culture. Sociol Res Online 2004;9:98-109.

2. Albrecht JS, Wickwire EM, Vadlamani A, Scharf SM, Tom SE. Trends in insomnia diagnosis and treatment among Medicare beneficiaries, 2006-2013. Am J Geriatr Psychiatry 2019;27:301-9.

3. Kronholm E, Partonen T, Laatikainen T, Peltonen M, Härmä M, Hublin C, et al. Trends in self-reported sleep duration and insomnia-related symptoms in Finland from 1972 to 2005: a comparative review and re-analysis of Finnish population samples. J Sleep Res 2008;17:54-62.

4. Matricciani L, Olds T, Petkov J. In search of lost sleep: secular trends in the sleep time of school-aged children and adolescents. Sleep Med Rev 2012;16:203-11.

5. Pallesen S, Sivertsen B, Nordhus IH, Bjorvatn B. A 10-year trend of insomnia prevalence in the adult Norwegian population. Sleep Med 2014; 15:173-9.

6. Ford ES, Wheaton AG, Cunningham TJ, Giles WH, Chapman DP, Croft JB. Trends in outpatient visits for insomnia, sleep apnea, and prescriptions for sleep medications among US adults: findings from the National Ambulatory Medical Care survey 1999-2010. Sleep 2014;37: 1283-93.

7. Ohayon MM. Epidemiology of insomnia: what we know and what we still need to learn. Sleep Med Rev 2002;6:97-111.

8. Fernandez-Mendoza J, Vgontzas AN. Insomnia and its impact on physical and mental health. Curr Psychiatry Rep 2013;15:418.

9. Barnes CM, Drake CL. Prioritizing sleep health: public health policy recommendations. Perspect Psychol Sci 2015;10:733-7.

10. Penzel T, Conradt R. Computer based sleep recording and analysis. Sleep Med Rev 2000;4:131-48.

11. Sadeh A. The role and validity of actigraphy in sleep medicine: an update. Sleep Med Rev 2011;15:259-67.

12. Martin JL, Hakim AD. Wrist actigraphy. Chest 2011;139:1514-27.

13. Kryger MH, Roth T, Dement WC. Principles and practice of sleep medicine. 6th ed. Philadelphia: Elsevier 2017;1671-8.

14. Van Someren EJ. Actigraphic monitoring of sleep and circadian rhythms. Handb Clin Neurol 2011;98:55-63.

15. Kryger MH, Roth T, Dement WC. Principles and practice of sleep medicine. Philadelphia: W.B. Saunders 2000;1295-301.

16. McPartland RJ, Kupfer DJ, Foster FG. The movement-activated recording monitor: a third-generation motor-activity monitoring system. Behav Res Methods 1976;8:357-60.

17. Blackwell T, Redline S, Ancoli-Israel S, Schneider JL, Surovec S, Johnson NL, et al. Comparison of sleep parameters from actigraphy and polysomnography in older women: the SOF study. Sleep 2008;31:28391.

18. Cellini N, Buman MP, McDevitt EA, Ricker AA, Mednick SC. Direct comparison of two actigraphy devices with polysomnographically recorded naps in healthy young adults. Chronobiol Int 2013;30:691-8.

19. Kosmadopoulos A, Sargent C, Darwent D, Zhou X, Roach GD. Alternatives to polysomnography (PSG): a validation of wrist actigraphy and a partial-PSG system. Behav Res Methods 2014;46:1032-41.

20. De Bellis N. Bibliometrics and citation analysis: from the science citation index to cybermetrics. Lanham: Scarecrow Press 2009.

21. Chang YW, Huang MH, Lin CW. Evolution of research subjects in library and information science based on keyword, bibliographical coupling, and co-citation analyses. Scientometrics 2015;105:2071-87.

22. Ohayon MM, Carskadon MA, Guilleminault C, Vitiello MV. Metaanalysis of quantitative sleep parameters from childhood to old age in healthy individuals: developing normative sleep values across the human lifespan. Sleep 2004;27:1255-73.

23. Lauderdale DS, Knutson KL, Yan LL, Liu K, Rathouz PJ. Self-reported and measured sleep duration: how similar are they? Epidemiology 2008; 19:838-45.

24. Sharma HP. Download plus citation counts-a useful indicator to mea- 
sure research impact. Curr Sci 2007;92:873.

25. Agarwal R, Light RP. The effect of measuring ambulatory blood pressure on nighttime sleep and daytime activity--implications for dipping. Clin J Am Soc Nephrol 2010;5:281-5.

26. Chen X, Wang R, Zee P, Lutsey PL, Javaheri S, Alcántara C, et al. Racial/ethnic differences in sleep disturbances: the Multi-Ethnic Study of Atherosclerosis (MESA). Sleep 2015;38:877-88.

27. Schwab KE, Ronish B, Needham DM, To AQ, Martin JL, Kamdar BB. Actigraphy to evaluate sleep in the intensive care unit. A systematic review. Ann Am Thorac Soc 2018;15:1075-82.

28. Qaseem A, Kansagara D, Forciea MA, Cooke M, Denberg TD. Management of chronic insomnia disorder in adults: a clinical practice guideline from the American College of Physicians. Ann Intern Med 2016;165: 125-33.

29. Riemann D, Baglioni C, Bassetti C, Bjorvatn B, Dolenc Groselj L, Ellis JG, et al. European guideline for the diagnosis and treatment of insomnia. J Sleep Res 2017;26:675-700.

30. Palermo TM, Beals-Erickson S, Bromberg M, Law E, Chen M. A single arm pilot trial of brief cognitive behavioral therapy for insomnia in adolescents with physical and psychiatric comorbidities. J Clin Sleep Med 2017;13:401-10.

31. Esaki Y, Takeuchi I, Tsuboi S, Fujita K, Iwata N, Kitajima T. A doubleblind, randomized, placebo-controlled trial of adjunctive blue-blocking glasses for the treatment of sleep and circadian rhythm in patients with bipolar disorder. Bipolar Disord 2020;22:739-48.

32. James SM, Honn KA, Gaddameedhi S, Van Dongen HPA. Shift work: disrupted circadian rhythms and sleep-implications for health and well-being. Curr Sleep Med Rep 2017;3:104-12.

33. Heath G, Dorrian J, Coates A. Associations between shift type, sleep, mood, and diet in a group of shift working nurses. Scand J Work Environ Health 2019;45:402-12.

34. Mizuno K, Matsumoto A, Aiba T, Abe T, Ohshima H, Takahashi M, et al. Sleep patterns among shift-working flight controllers of the International Space Station: an observational study on the JAXA Flight Control Team. J Physiol Anthropol 2016;35:19.

35. Mendonça F, Mostafa SS, Morgado-Dias F, Ravelo-Garcia AG, Penzel T. A review of approaches for sleep quality analysis. IEEE Access 2019; 7:24527-46.

36. Schoch SF, Kurth S, Werner H. Actigraphy in sleep research with infants and young children: current practices and future benefits of standardized reporting. J Sleep Res 2021;30:e13134.

37. Ellegaard O, Wallin JA. The bibliometric analysis of scholarly production: how great is the impact? Scientometrics 2015;105:1809-31.

38. Lee K, Jung H. Dynamic semantic network analysis for identifying the concept and scope of social sustainability. J Clean Prod 2019;233:151024.

39. Kim SH, Chang RS. The study on the research trend of social network analysis and the its applicability to information science. JKOSIM 2010; 27:71-87.

40. Kho J, Cho K, Cho Y. A study on recent research trend in management of technology using keywords network analysis. J Intell Inform Syst 2013;19:101-23. 